Bull. Austral. Math. Soc.

Vol. 38 (1988) [1-10]

\title{
QUADRATIC SYSTEMS \\ WITH A DEGENERATE CRITICAL POINT
}

W. A. Coppel,

It is shown that a quadratic system with a degenerate critical point has at most one limit cycle.

A critical point of a two-dimensional autonomous system

$$
x^{\prime}=P(x, y), \quad y^{\prime}=Q(x, y),
$$

is a point $M=\left(x_{0}, y_{0}\right)$ for which

$$
P\left(x_{0}, y_{0}\right)=Q\left(x_{0}, y_{0}\right)=0
$$

The critical point is degenerate if both the Jacobian $P_{x} Q_{y}-P_{y} Q_{x}$ and the divergence $P_{x}+Q_{y}$ are zero at $M$.

The system (1) is said to be quadratic if

$$
P(x, y)=\sum_{i+k=0}^{2} a_{i k} x^{i} y^{k} \quad Q(x, y)=\sum_{i+k=0}^{2} b_{i k} x^{i} y^{k}
$$

are relatively prime real polynomials of degree at most two which are not both linear. We propose to prove

THEOREM 1. If a quadratic system has a degenerate critical point then it either has a centre or has at most one periodic orbit. Moreover, if there is a unique periodic orbit it is a limit cycle with a non-zero characteristic exponent.

By [2], Theorem 6 we may suppose that the quadratic system has a focus or centre at the origin, which is surrounded by a periodic orbit. Moreover, by a non-singular linear transformation we may suppose that it has the form

$$
\begin{aligned}
& x^{\prime}=\lambda x-y+l x^{2}+m x y+n y^{2} \\
& y^{\prime}=x+\lambda y+a x^{2}+b x y+c y^{2} .
\end{aligned}
$$

Received 1 September 1987

Copyright Clearance Centre, Inc. Serial-fee code: 0004-9729/88 \$A2.00+0.00. 
Furthermore we may suppose that the degenerate critical point is situated at $(0,1)$. Then $n=1, c=-\lambda$ and $m=0, b=-1-\lambda^{2}$. Thus

$$
\begin{aligned}
& x^{\prime}=\lambda x-y+l x^{2}+y^{2} \\
& y^{\prime}=x+\lambda y+a x^{2}-\left(1+\lambda^{2}\right) x y-\lambda y^{2} .
\end{aligned}
$$

If we put $z=y+\lambda x$ this takes the form

$$
\begin{aligned}
& x^{\prime}=2 \lambda x-z+L x^{2}-2 \lambda x z+z^{2} \\
& z^{\prime}=\left(1+\lambda^{2}\right) x+A x^{2}-\left(1+\lambda^{2}\right) x y .
\end{aligned}
$$

By scaling we can obtain finally a system of the form

$$
\begin{aligned}
& x^{\prime}=d x-y+l x^{2}-d x y+y^{2} \\
& y^{\prime}=x+a x^{2}-x y
\end{aligned}
$$

where $-2<d<2$.

Since $y^{\prime}=a x^{2}$ for $y=1$ any periodic orbit $\gamma$ of (3) which surrounds the origin must lie in the half-plane $y<1$. Moreover, since the interior of $\gamma$ is a convex region ([2, Theorem 1]), the intersections of $\gamma$ with the $y$-axis are the only points on $\gamma$ where $y^{\prime}=0$. Hence $\gamma$ must also lie in the half-plane $1+a x-y>0$.

The system (3) has a centre at the origin if $d=a(2 l-1)=0$, by the standard criteria for a weak focus to be a centre (see [2]). Thus this case may be excluded. If we set $B(y)=|1-y|^{\alpha}$ then for the system (3)

$$
(B P)_{x}+(B Q)_{y}=\left[d(1-y)^{2}+(2 l-1-\alpha) x(1-y)-\alpha a x^{2}\right] B /(1-y) .
$$

If we take $\alpha=2 l-1$ then it follows from Dulac's criterion that $a d(2 l-1)>0$. If we take $\alpha=1-2 l$ then it follows similarly that $(2 l-1)^{2}>a d(2 l-1)$. By changing the signs of $x$ and $t$ we may suppose that $d>0$. Then we must have

$$
0<d<(2 l-1) / a \text {. }
$$

It will now be shown that the quadratic system (3) has no focus or centre besides the origin, and hence that every periodic orbit must surround the origin. Besides the critical points $(0,0)$ and $(0,1)$ the system $(3)$ also has the critical point $\left(x_{0}, y_{0}\right)$, where

$$
x_{0}=-a /\left(a^{2}-a d+l\right), \quad y_{0}=1+a x_{0}=(l-a d) /\left(a^{2}-a d+l\right) .
$$

If we set $x=x_{0}+\xi, y=y_{0}+\eta$ then

$$
\begin{aligned}
\xi^{\prime} & =(2 l-a d) x_{0} \xi+\left[1+(2 a-d) x_{0}\right] \eta+\ldots \\
\eta^{\prime} & =a x_{0} \xi-x_{0} \eta+\ldots
\end{aligned}
$$


Thus the linear terms have trace $T=(2 l-a d-1) x_{0}$ and determinant $D=a x_{0}$. If the critical point $\left(x_{0}, y_{0}\right)$ is a focus or centre then $T^{2}<4 D$, that is

$$
(2 l-1-a d)^{2}<-4\left(a^{2}-a d+l\right)
$$

or, equivalently,

$$
(2 l+1-a d)^{2}<4\left(l-a^{2}\right) .
$$

Thus $a^{2}-a d+l<0$ and $l>a^{2}$. Since $d>0$, these inequalities imply $a>0$. Then (4) implies $2 l+1-a d>2$ and hence $1<l-a^{2}$. On the other hand $a^{2}-a d+1>0$, since $0<d<2$, and hence $l<1$. Thus we have a contradiction.

In order to show that the quadratic system (3) has at most one periodic orbit surrounding the origin we now make some changes of variables. The linear fractional transformation

$$
\xi=x /(1-y), \eta=y /(1-y), d \tau / d t=1-y
$$

with inverse

$$
x=\xi /(1+\eta), y=\eta /(1+\eta), d t / d \tau=1+\eta
$$

replaces (3) by

$$
\begin{aligned}
& \xi^{\prime}=d \xi+(1+l) \xi^{2}+a \xi^{3}-\eta \\
& \eta^{\prime}=\left(\xi+a \xi^{2}\right)(1+\eta)
\end{aligned}
$$

We wish to show that this system has at most one periodic orbit in the region $\eta>$ $-1,1+a \xi>0$.

If we now put $\xi=x, \eta=e^{y}-1, \tau=t$ we obtain a system

$$
\begin{aligned}
& x^{\prime}=F(x)-\varphi(y) \\
& y^{\prime}=g(x)
\end{aligned}
$$

with

$$
\begin{aligned}
& F(x)=d x+(1+l) x^{2}+a x^{3} \\
& g(x)=x+a x^{2} \\
& \varphi(y)=e^{y}-1
\end{aligned}
$$

In order to show that (5)-(6) has at most one periodic orbit in the region $1+a x>0$ we make use of the following criterion: 
TheOREM 2. Let $\varphi$ be a continuously differentiable function such that $\varphi(0)=$ $0, \varphi^{\prime}(y)>0$ for $-\infty<y<\infty$. Also let $f, g$ be continuously differentiable functions on the open interval $(a, b)$, where $a<0<b$, such that

(i) $g(x) \geqslant 0$ according as $x \gtrless 0$,

(ii) $f(0)>0$,

(iii) $f(x) / g(x)$ is a decreasing function for $x>0$ and for $x<0$.

Then the system (5), where $F(x)=\int_{0}^{x} f(\xi) d \xi$, has at most one periodic orbit and, if it exists, it is a stable limit cycle.

Theorem 2 differs only trivially from the theorem in Zhang Zhifen [5] and may be proved in exactly the same way. (This theorem was already announced, with a sketch of the proof, in [4].) It will now be shown that the functions (6) satisfy the conditions of Theorem 2. The assumptions concerning $\varphi$ are obviously satisfied. The hypothesis (i) is satisfied on the open interval where $1+a x>0$ and the hypothesis (ii) is also satisfied, since

$$
f(x)=d+2(1+l) x+3 a x^{2} .
$$

Finally the hypothesis (iii) is satisfied, since

$$
\frac{d}{d x}(f / g)=-\left[d+2 a d x+a(2 l-1) x^{2}\right] / g^{2}(x)
$$

and the quadratic in square brackets has negative discriminant.

Hence the quadratic system (3) has a unique periodic orbit, which is a stable limit cycle. To complete the proof of Theorem 1 it only remains to show that this periodic orbit has a negative characteristic exponent.

Let $\gamma$ now denote the unique periodic orbit of the system (5)-(6). Let $x_{2}>0$ be the maximum value of $x$ on $\gamma$ and put

$$
\bar{f}(x)=f(x)-\alpha g(x)
$$

where $\alpha=f\left(x_{2}\right) / g\left(x_{2}\right)$. The hypotheses (i)-(iii) of Theorem 2 imply that $\alpha>$ $0, \bar{f}(x)<0$ for $x>x_{2}$ and $\bar{f}(x)>0$ for $0 \leqslant x<x_{2}$. The characteristic exponent of $\gamma$ is

$$
h=\int_{\gamma} \bar{f}(x) d t
$$

since

$$
\int_{\gamma} g(x) d t=\int_{\gamma} d y=0
$$

Since $\gamma$ is stable we must have $h \leqslant 0$. We will assume $h=0$ and derive a contradiction. 
Evidently $\bar{f}\left(x_{1}\right)=0$ for some $x_{1}<0$ and the minimum value of $x$ on $\gamma$ is less than $x_{1}$. The hypotheses (i)-(iii) of Theorem 2 imply that $\bar{f}(x)<0$ for $x<x_{1}$ and $\bar{f}(x)>0$ for $x_{1}<x<0$. Put

$$
\Psi(x)= \begin{cases}\exp & {\left[-\left(x_{1}-x\right)^{-2}\right] \text { for } x<x_{1}} \\ 0 & \text { for } x \geqslant x_{1}\end{cases}
$$

and set

$$
f_{\mu}(x)=f(x)+\mu \Psi(x) g(x)
$$

where $\mu>0$. Then the corresponding system

$$
\begin{aligned}
& x^{\prime}=F_{\mu}(x)-\varphi(y) \\
& y^{\prime}=g(x)
\end{aligned}
$$

also satisfies the hypotheses of Theorem 2. Moreover $\left(5_{\mu}\right)$ has a periodic orbit $\gamma_{\mu}$ in the interior of $\gamma$, since the origin is an unstable critical point and orbits of $\left(5_{\mu}\right)$ which intersect $\gamma$ to the left of the line $x=x_{1}$ cross it from the outside to the inside. Thus $\gamma_{\mu}$ is the unique periodic orbit of $\left(5_{\mu}\right)$ and is a stable limit cycle.

We now make use of some results from Andronov et al. [1, Chapter 13]. Although the system $\left(5_{\mu}\right)$ is $C^{\infty}$, rather than analytic, and although it is not obtained from the analytic system (5) by a simple rotation of the vector field, nevertheless the quantity $u_{01}$ defined there by $(36)$ is still non-zero. Consequently Theorems 71 and 72 continue to hold. Theorem 71 shows that the limit cycle $\gamma$ of (5) must be of odd multiplicity. Theorem 72 then shows that for all sufficiently small $\mu>0$ the limit cycle $\gamma_{\mu}$ of $\left(5_{\mu}\right)$ has characteristic exponent $h_{\mu}<0$.

Finally we compare $h_{\mu}$ with $h$, in the same manner as in the proof of Theorem 2. We refer to Figure 1 . Since $\gamma$ is a limit cycle of odd multiplicity greater than 1 , the argument in [1] shows that the distance $\sigma$ of $B_{1}$ from $A_{0}$ is given asymptotically by $\sigma \sim c_{1} \mu^{\alpha}$ for $\mu \rightarrow+0$, where $c_{1}>0$ and $0<\alpha \leqslant 1 / 3$. Evidently

$$
J_{1}=\int_{A_{1} B_{1}} \bar{f}(x) d t<0, \quad J_{2}=\int_{C_{1} D_{1}} \bar{f}(x) d t<0 .
$$

Since $x^{\prime}<0$ along $F_{1} A_{1}$ and $C_{0} A_{0}$ these arcs can be described by equations $y=y(x)$ and $y=y_{\mu}(x)$ respectively. Then 


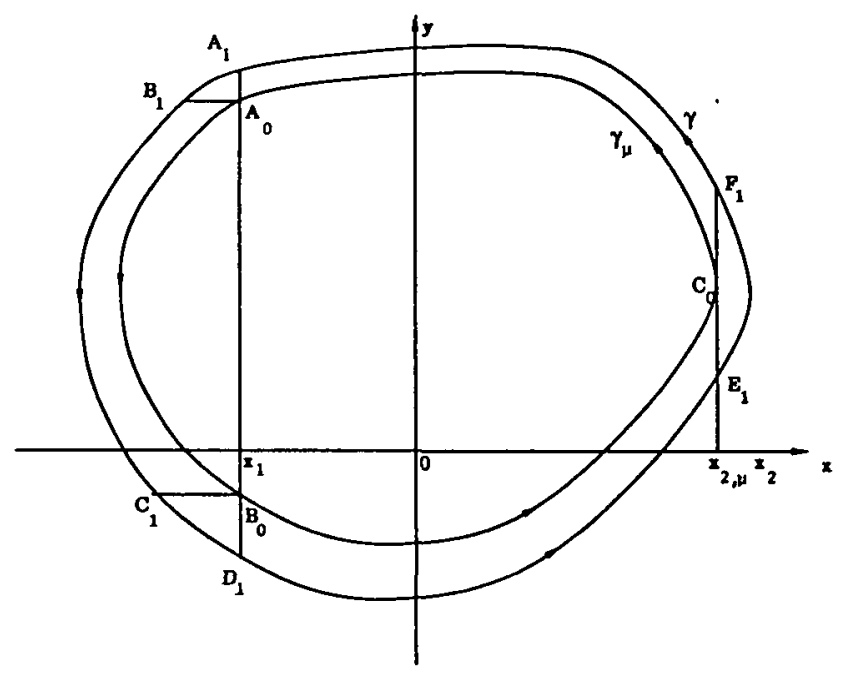

Figure 1

$$
\begin{aligned}
J_{3} & =\int_{F_{1} A_{1}} \bar{f}(x) d t-\int_{C_{0} A_{0}} \bar{f}_{\mu}(x) d t \\
& =-\int_{x_{1}}^{x_{2, \mu}} \frac{\bar{f}(x) d x}{F(x)-\varphi(y(x))}+\int_{x_{1}}^{x_{2, \mu}} \frac{\bar{f}(x) d x}{F(x)-\varphi\left(y_{\mu}(x)\right)} \\
& =\int_{x_{1}}^{x_{2, \mu}} \frac{\bar{f}(x)\left[\varphi\left(y_{\mu}(x)\right)-\varphi(y(x))\right] d x}{[F(x)-\varphi(y(x))]\left[F(x)-\varphi\left(y_{\mu}(x)\right)\right]} \\
& <0
\end{aligned}
$$

since $y_{\mu}(x)<y(x)$ for $x_{1}<x<x_{2, \mu}$. Similarly

$$
J_{4}=\int_{D_{1} E_{1}} \bar{f}(x) d t-\int_{B_{0} C_{0}} \bar{f}_{\mu}(x) d t<0 .
$$

Since $y^{\prime}<0$ along $B_{1} C_{1}$ and $A_{0} B_{0}$ these arcs can be described by equations $x=x(y)$ and $x=x_{\mu}(y)$ respectively. Let $y_{1}, y_{2}\left(y_{1}<0<y_{2}\right)$ be the ordinates of $B_{0}$ and $A_{0}$. Then since $(f / g)^{\prime}<0$ and $\alpha<1$ we have, for small $\mu>0$,

$$
\begin{aligned}
J_{5} & =\int_{B_{1} C_{1}} \bar{f}(x) d t-\int_{A_{0} B_{0}} \bar{f}_{\mu}(x) d t \\
& =\int_{y_{1}}^{y_{2}}\left[\frac{f\left(x_{\mu}(y)\right)}{g\left(x_{\mu}(y)\right)}-\frac{f(x(y))}{g(x(y))}\right] d y+\mu \int_{y_{1}}^{y_{2}} \Psi\left(x_{\mu}(y)\right) d y \\
& <-c_{2} \mu^{\alpha}
\end{aligned}
$$


where $c_{2}>0$. On the arc $E_{1} F_{1}$ we have $x=\tilde{x}(y)$. Since $\bar{f}\left(x_{2}\right)=0$ it follows that

$$
J_{6}=\int_{E_{1} F_{1}} \bar{f}(x) d t=\int \frac{\bar{f}(\tilde{x}(y))}{g(\tilde{x}(y))} d y=o\left(\mu^{\alpha}\right) .
$$

Adding these estimates we obtain, for small $\mu>0$,

$$
h-h_{\mu}=J_{1}+J_{2}+J_{3}+J_{4}+J_{5}+J_{6}<0
$$

Since $h=0$ and $h_{\mu}<0$, this is the required contradiction. This completes the proof of Theorem 1.

As Poincaré already pointed out, it is useful to extend the domain of definition of a quadratic system to the real projective plane. Then, in addition to the finite critical points, there are also critical points at infinity. In [3] I have shown that if a quadratic system has a critical point at infinity which is degenerate then it either has a centre or has at most one periodic orbit. Moreover, if there is a unique periodic orbit it is a limit cycle with a non-zero characteristic exponent. Thus the statement of Theorem 1 may be understood to refer to either a finite or an infinite critical point.

The proof given in [3] for a degenerate critical point at infinity depended on comparing the quadratic system with certain reflected systems and on the fact that the vector field defined by a quadratic system has at most six points of contact with a conic section, unless the conic section is invariant. In the same paper it was shown by a quite different method that a quadratic system with an invariant line has at most one limit cycle. That proof depended on the following uniqueness theorem for the Liénard equation

$$
x^{\prime \prime}-f(x) x^{\prime}+g(x)=0
$$

or rather the equivalent system

$$
\begin{aligned}
& x^{\prime}=F(x)-y \\
& y^{\prime}=g(x)
\end{aligned}
$$

where

$$
F(x)=\int_{0}^{x} f(\xi) d \xi
$$

ThEOREM 3. Let $f, g$ be continuously differentiable functions on the open interval $(a, b)$, where $a<0<b$, such that

(i) $g(x) \geqslant 0$ according as $x \geqslant 0$, 
(ii) $f(x) \gtrless 0$ according as $x \gtrless x_{0}$, where $x_{0}<0$,

(iii) the simultaneous equations

$$
F\left(x_{1}\right)=F\left(x_{2}\right), f\left(x_{1}\right) / g\left(x_{1}\right)=f\left(x_{2}\right) / g\left(x_{2}\right)
$$

have at most one solution $x_{1}, x_{2}$ with $a<x_{1}<x_{0}$ and $0<x_{2}<b$.

(iv) if $F\left(\xi_{0}\right)=0$ for some $\xi_{0}<x_{0}$ then $f(x) F(x) / g(x)$ is a decreasing function for $a<x<\xi_{0}$.

Then the system (8) has at most one periodic orbit and, if it exists, it has a negative characteristic exponent.

If in the proof of Theorem 3 given in [3] we replace the path $\bar{\gamma}$ through the point $C$ by the path $\bar{\gamma}$ through the point $D$ then in a completely analogous way we can prove

TheOREM $3^{\prime}$. The conclusions of Theorem 3 still hold if the hypotheses (i)-(iii) are retained but the hypothesis (iv) is replaced by

(iv) $f(x) F(x) / g(x)$ is an increasing function for $0<x<b$ and

$$
\lim _{x \rightarrow a+} F(x) \leqslant \lim _{x \rightarrow b-} F(x) .
$$

In conclusion we show that Theorem 1, for the case of a degenerate critical point at infinity, can actually be deduced from Theorem $3^{\prime}$. Proceeding as in [3], we see that it is sufficient to show that the quadratic system

$$
\begin{aligned}
& x^{\prime}=y+c x^{2} \\
& y^{\prime}=-x+d y+n x^{2}+m x y
\end{aligned}
$$

where $d>0, n<(3 c-1) d$ and $m+2 c=1$, has at most one periodic orbit surrounding the origin. Eliminating $y$ we obtain a Liénard equation (7) with

$$
f(x)=d+x, g(x)=x q(x)
$$

where

$$
q(x)=1+(c d-n) x+c(1-2 c) x^{2} .
$$

We will show that the hypotheses of Theorem $3^{\prime}$ are satisfied by this Liénard equation.

Let $\gamma$ be a periodic orbit of (8) which surrounds the origin. If $q(\xi)=0$ then on the line $x=\xi x^{\prime}$ has opposite signs on opposite sides of the critical point $\left(\xi,-c \xi^{2}\right)$. Since the interior of $\gamma$ is a convex region containing no critical point besides the origin it follows that $\gamma$ cannot intersect the line $x=\xi$. We now construct an open interval 
$(a, b)$ with $a<0<b$, such that $q(x)>0$ for $a<x<b$ and any periodic orbit of (8) which surrounds the origin must lie in the strip $a<x<b$. Then the hypothesis (i) will be satisfied.

If $q(x)$ has no real zeros we take $a=-\infty, b=+\infty$. If $q(x)$ has two real zeros $\xi_{1}<\xi_{2}<0$, or if $q(x)$ has only one zero $\xi_{2}$ and $\xi_{2}<0$, we take $a=\xi_{2}, b=+\infty$. If $0 \leqslant c \leqslant 1 / 2$ then one of the preceding possibilities must occur, since $n-c d<0$ and $c(1-2 c) \geqslant 0$. If $c<0$ or if $c>1 / 2$ then $q(x)$ has two real zeros $\xi_{1}<0<\xi_{2}$ and we take $a=\xi_{1}, b=\xi_{2}$.

If $-d \leqslant a$ the system (8) has no periodic orbits, since the divergence $f(x)$ is of constant sign for $a<x<b$. Hence we may suppose $a<-d$ and then the hypothesis (ii) is obviously satisfied with $x_{0}=-d$.

Since $F(x)=d x+x^{2} / 2$ it is readily shown that the simultaneous equations (9) with $x_{1}<0<x_{2}$ are equivalent to

$$
x_{1}+x_{2}=-2 d, d q(-2 d)=H x_{1} x_{2}
$$

where

$$
H=n-c d+3 c d(1-2 c)
$$

Since $a<x_{1}<-2 d$ we must have $q(-2 d)>0$ and since $x_{1} x_{2}<0$ we must have $H<0$. Since the first equation (11) defines $x_{2}$ as a decreasing function of $x_{1}$ and the second equation (11) defines $x_{2}$ as an increasing function of $x_{1}$, it follows that the hypothesis (iii) is also satisfied.

If we put $\omega(x)=f(x) F(x) / g(x)$ then

$$
\begin{aligned}
\omega^{\prime}(x) / \omega(x) & =\frac{1}{d+x}+\frac{1}{2 d+x}-\frac{q^{\prime}(x)}{q(x)} \\
& =N(x) /(d+x)(2 d+x) q(x)
\end{aligned}
$$

where

$$
N(x)=3 d+2 d^{2}(n-c d)+2\left[1-2 c(1-2 c) d^{2}\right] x-H x^{2} .
$$

Since $N(-d)=d q(-d)>0, N(-2 d)=-d q(-2 d)<0$ and $N(-\infty)=+\infty$, the quadratic $N(x)$ is positive for $x>-d$ and in particular for $x>0$. It follows that $\omega(x)$ is an increasing function for $0<x<b$. The second part of the hypothesis (iv)' is also satisfied, since if $c<0$ or $c>1 / 2$ then

$$
a+b=\frac{n-c d}{c(1-2 c)}>-d / c>-2 d
$$

Thus we have a new proof of Theorem 1 when the degenerate critical point is at infinity. 


\section{REFERENCES}

[1] A.A. Andronov, E.A. Leontovich, I.I. Gordon and A.G. Maier, Theory of bifurcations of dynamic systems on a plane (Halsted Press, New York - Toronto, 1973).

[2] W.A. Coppel, 'A survey of quadratic systems', J. Differential Equations 2 (1966), 293-304.

[3] W.A. Coppel, 'Some quadratic systems with at most one limit cycle', Dynamics Reported (to appear).

[4] Zhang Zhifen (Chan Chi-fen), 'On the uniqueness of limit cycles of certain equations of nonlinear oscillations', Dokl. Akad. Nauk SSSR 110 (1958), 659-662. [Russian] .

[b] Zhang Zhifen, 'Proof of the uniqueness theorem of limit cycles of generalized Liénard equations', Applicable Anal. 23 (1986), 63-76.

Department of Mathematics, I.A.S.

Australian National University

G.P.O. Box 4, Canberra, A.C.T. 2601

Australia 